\title{
步长加速法优化 $\mathbf{B}$ 样条参数的离散数据点拟合
}

\author{
张莉 ${ }^{1)}$, 张能俊 ${ }^{1)}$, 姚红丽 ${ }^{1)}$, 檀结庆 ${ }^{1,2)}$ \\ ${ }^{1)}$ (合肥工业大学数学学院 合肥 230009) \\ ${ }^{2)}$ (合肥工业大学计算机与信息学院 合肥 230009) \\ (lizhang@hfut.edu.cn)
}

摘 要: 采用迭代法拟合离散数据点时, 数据点的参数化会同时影响逼近的效果和逼近的速度, 为此, 提出一种通 过迭代调整优化控制顶点和数据点参数的方法, 其收玫速度较快且拟合得到曲线更贴合控制点. 首先, 选取初始控 制顶点，通过自适应的 BFGS 方法优化控制顶点得到拟合曲线; 其次，保持控制顶点不变，利用步长加速法优化数据 点对应的参数; 最后, 利用新参数值重新优化控制顶点并得到新的拟合曲线. 数值实例表明, 所提方法在迭代前期 步骤中，收敛速度快于现有的基于控制顶点迭代法，且优化后的曲线更加逼近离散的数据点，拟合误差更小.

关键词：无约束优化; BFGS 方法; B 样条; 步长加速法; 曲线拟合

中图法分类号: TP391.41

DOI: $10.3724 /$ SP.J.1089.2021.18414

\section{Discrete Data Points Fitting Based on Optimization of B-Spline Parameters Using Step-Acceleration Method}

\author{
Zhang $\mathrm{Li}^{1)}$, Zhang Nengjun ${ }^{1)}$, Yao Hongli ${ }^{1)}$, and Tan Jieqing ${ }^{1,2)}$ \\ ${ }^{1)}$ (School of Mathematics, Hefei University of Technology, Hefei 230009) \\ 2) (School of Computer and Information, Hefei University of Technology, Hefei 230009)
}

\begin{abstract}
When fitting discrete data points by iterative method, the parameterization of data points will affect the approximation effect and speed at the same time. A method of optimizing the parameters of control vertices and data points by iterative adjustment is proposed, which converges faster and fits the original data points better. Firstly, the initial control vertices are selected, and the adaptive BFGS method is used to optimize the control vertices and obtain the fitting curve. Secondly, the parameters corresponding to data points are optimized by the step-size acceleration method while the control vertices are kept unchanged. Finally, the new parameters are used to re-optimize the control vertices and a new fitting curve is obtained. Numerical examples show that the convergence speed in the early iteration stage of the given algorithm is faster than most existing iterative methods. Furthermore, the optimized curves are much closer to discrete data points and fitting error are much smaller.
\end{abstract}

Key words: unconstrained optimization; BFGS method; B-spline; step-size acceleration; curve fitting

计算机图形学 ${ }^{[1]}$ 、计算机辅助设计 ${ }^{[2-3]}$ 、逆向 工程和数据可视化等多个科学技术领域都需要对 数据点进行处理, 采用的方法可分为插值和拟合.
由于采样或实验得到的数据点存在舍人误差、分布 不规律等问题，在实际应用中，常采用拟合的方 法. 由于 B 样条曲线具有良好的几何性质, 因此其

收稿日期：2020-05-31; 修回日期：2020-09-24. 基金项目：国家自然科学基金(61972131); 国家重点研发计划(2018YFB2100301). 张莉(1976-), 女, 博士, 教授, 博士生导师, CCF 会员, 主要研究方向为计算机辅助几何设计; 张能俊(1995一), 男, 硕士研究生, 主 要研究方向为计算机辅助几何设计; 姚红丽(1997-), 女, 硕士研究生, 主要研究方向为计算机辅助几何设计; 檀结庆(1962一), 男, 博士, 教授, 博士生导师, 主要研究方向为非线性数值逼近理论与方法、科学计算可视化、计算机辅助几何设计和图形学、图像处理. 
在数据点拟合中广泛应用. 其中影响 B 样条曲线 拟合效果的因素包括控制顶点、数据点参数化的方 法以及节点向量的选择.

渐进迭代逼近(progressive iterative approximation, PIA)是一种具有明显几何意义的迭代方法, 通过迭代调整控制顶点，使得到的极限曲线/曲面 插值给定的数据点. PIA 方法起源于齐东旭等 ${ }^{[4]}$ 和 de Boor ${ }^{[5]}$ 对均匀三次 B 样条的递进迭代研究. 2003 年, 萄宏伟等 ${ }^{[6]}$ 提出非均匀三次 $\mathrm{B}$ 样条曲线拟合也 具有 PIA 的性质，并将该方法推广到全正基的混 合曲线曲面上 ${ }^{[7]} . \mathrm{Lu}^{[8]}$ 提出带权值的渐进迭代逼近 方法(weighted progressive iterative approximation, WPIA)，并给出了最优权值的取法. 由于上述 PIA 方法中控制顶点的数目等于数据点的数目, 因此 并不适用于大规模的数据点拟合. 针对这个问题, Lin 等 ${ }^{[9]}$ 提出了推广的 PIA(extended progressive iterative approximation, EPIA)，允许控制顶点选取 数目少于数据点数目, 可以有效地减少计算量, 提 高计算效率. 2014 年, Deng 等 ${ }^{[10]}$ 提出基于最小二乘 的渐进迭代逼近方法(progressive iterative approximation for least squares, LSPIA)，与传统的最小二乘方 法相比, LSPIA 不仅具备传统 PIA 的优良性质, 还可 以高效、直观地处理大规模数据点集. Zhang 等 ${ }^{[11-12]}$ 研究了广义 B 样条的 LSPIA 算法, 并给出 2 种不同 的权值, 通过权值的调整与核函数的合适选取加 快基于广义 $\mathrm{B}$ 样条算法的收敛速度. 在传统的 PIA 方法中, 数据点对应的参数值是不变的. 日本学者 Maekawa 等 ${ }^{[13]}$ 提出几何插值(geometric interpolation, $\mathrm{GI}$ )方法，将每次迭代生成的拟合曲线曲面上最近 点的参数值选取为对应数据点的参数. 由于 PIA 和 GI 方法都具有明显的几何意义，文献[14-15]将 这些几何意义明显的迭代法统称为几何迭代法, 对其进行了全面总结，系统地阐述了几何迭代法 的思想和应用.

除了对 $\mathrm{B}$ 样条曲线控制顶点的调整可以减少 拟合误差，选取合适的参数化方法和节点向量也 可以减少拟合误差. 有大量文献 ${ }^{[16-19]}$ 对此进行了 研究, 现有参数的选择包括均匀参数化法、累加弦 长法、向心参数法, 也可以通过一些其他方法对参 数进行调整. Speer 等 ${ }^{[16]}$ 利用 Gauss-Newton 方法同 时优化控制点和数据点的参数, 但是这种梯度法 存在一些缺点, 如依赖初始值的选取, 得到的优化 参数值不是按序递增. 周明华等 ${ }^{\left[{ }^{[7]}\right.}$ 用遗传算法同 时优化参数和节点, 使拟合结果在最小二乘拟合误 差下最小. $\mathrm{Hu}$ 等 ${ }^{[18]}$ 提出一种非均匀 $\mathrm{B}$ 样条曲线曲面
的 PIA 优化算法, 通过遗传算法优化参数和节点, 有效地减少插值曲线曲面的形状扭曲. 张蒙等 ${ }^{[19]}$ 利 用二分法分别优化 NURBS 曲线的数据点参数、节 点和权因子，逐个、全面地优化各参数，提高拟合 精度. 李莎莎等 ${ }^{[20]}$ 通过对数据点加权, 提出一种插 值部分数据点的最小二乘渐进迭代逼近方法.

本文将数据点拟合问题转化为求解无约束优 化问题, 对控制顶点和数据点参数分别进行优化. 拟牛顿法是一种求解无约束最小化问题的有效方 法, 其中, BFGS 方法 ${ }^{[21-24]}$ 是应用最广泛的拟牛顿 法之一. 一方面, 为了避免求解线性方程组并且提 高算法性能, 本文对 BFGS 方法进行改进, 通过加 人缩放参数，构造一种自适应的 BFGS 方法来优化 控制顶点; 另一方面, 为了保证优化的参数是按序 递增并且收玫的，本文利用步长加速法这种避免 梯度的直接法对参数逐个调整. 数值实例表明, 与 现有的几种调整控制点的方法相比，本文方法在 前几步迭代中收玫速度更快, 而通过对参数进行 优化使得到的拟合曲线更加接近数据点, 拟合误 差更小.

\section{1 预备知识}

首先介绍 $\mathrm{B}$ 样条基函数及 $\mathrm{B}$ 样条曲线的表示. 设 $U=\left\{u_{0}, \cdots, u_{m}\right\}$ 是非递减实数序列, 即 $u_{i} \leqslant$ $u_{i+1}, i=0, \cdots, m-1 . \quad u_{i}$ 称为节点, $U$ 称为节点序 列. 第 $i$ 个 $k$ 次 B 样条基函数 $N_{i}^{k}(u)$ 定义为

$$
\left\{\begin{array}{l}
N_{i}^{0}(u)=\left\{\begin{array}{l}
1, \text { if } u \in\left[u_{i}, u_{i+1}\right) \\
0, \text { otherwise }
\end{array}\right. \\
N_{i}^{k}(u)=\frac{u-u_{i}}{u_{i+k-1}-u_{i}} N_{i}^{k-1}(u)+\frac{u_{i+k}-u}{u_{i+k}-u_{i+1}} N_{i+1}^{k-1}(u)
\end{array} .\right.
$$

规定出现 $0 / 0$ 的情况时, 式中分式的比值取为 0 . 给定三维欧几里得空间 $\mathbb{R}^{3}$ 中的 $n$ 个点 $\left\{P_{i}\right\}_{i=0}^{n}$, 其 中 $P_{i}$ 表示控制顶点, 则对应节点序列 $U$ 的 $k$ 次 $\mathrm{B}$ 样条曲线表示为 $C(u)=\sum_{i=0}^{n} N_{i}^{k}(u) P_{i}$.

由于 $\mathrm{B}$ 样条基函数具有分段连续性和局部支 撑性，可以有效地应用于数据点的拟合中.

对于数据点的拟合，常采用最小二乘拟合方 法. 给定一组数据点 $\left\{Q_{j}\right\}_{j=0}^{m},\left\{P_{i}\right\}_{i=0}^{n}$ 表示控制顶 点 ( $m, n$ 为正整数). 数据点对应的参数表示为 $\left\{0=t_{0}<t_{1}<\cdots<t_{m}=1\right\}, \mathrm{B}$ 样条曲线可表示为 


$$
C(t)=\sum_{i=0}^{n} N_{i}(t) P_{i}=N(t) \boldsymbol{P}, t \in\left[t_{0}, t_{m}\right] .
$$

其中, $\left\{N_{i}(t), i=0,1, \cdots, n\right\}$ 表示 B 样条基; $N(t)=$ $\left(N_{0}(t), N_{1}(t), \cdots, N_{n}(t)\right) ; \boldsymbol{P}=\left(P_{0}, P_{1}, \cdots, P_{n}\right)^{\mathrm{T}}$.

设数据点与拟合曲线的误差函数为

$$
f=\sum_{j=0}^{m}\left(Q_{j}-C\left(t_{j}\right)\right)^{2}=\sum_{j=0}^{m}\left(Q_{j}-\boldsymbol{N}\left(t_{j}\right) \boldsymbol{P}\right)^{2}
$$

式(1)对应的矩阵形式为

$$
f=(\boldsymbol{Q}-\boldsymbol{N P})^{\mathrm{T}}(\boldsymbol{Q}-\boldsymbol{N P})
$$

其中, $Q_{0}=P_{0}, Q_{m}=P_{n} ; \boldsymbol{N}$ 表示在参数点 $\left\{t_{0}<\right.$ $\left.t_{1}<\cdots<t_{m-1}<t_{m}\right\}$ 处的配置矩阵, 即

$$
\boldsymbol{N}=\left[\begin{array}{cccc}
N_{0}\left(t_{0}\right) & N_{1}\left(t_{0}\right) & \cdots & N_{n}\left(t_{0}\right) \\
N_{0}\left(t_{1}\right) & N_{1}\left(t_{1}\right) & \cdots & N_{n}\left(t_{1}\right) \\
\cdots & \cdots & \cdots & \cdots \\
N_{0}\left(t_{m}\right) & N_{1}\left(t_{m}\right) & \cdots & N_{n}\left(t_{m}\right)
\end{array}\right] .
$$

最小二乘拟合方法的思想为: 将误差函数 $f$ 作为目标函数, 对目标函数求导, 得到一个关于未 知量为控制顶点的线性方程组, 通过求解线性方 程组得到拟合曲线. 由最小二乘法可得解为

$$
\boldsymbol{P}=\boldsymbol{N}^{-} \boldsymbol{Q} .
$$

其中, $\boldsymbol{N}^{-}$为 $(n+1) \times(m+1)$ 广义逆矩阵, 且满足

$$
\boldsymbol{N} \boldsymbol{N}^{-} \boldsymbol{N}=\boldsymbol{N},\left(\boldsymbol{N \boldsymbol { N } ^ { - }}\right)^{\mathrm{T}}=\boldsymbol{N} \boldsymbol{N}^{-} .
$$

在拟合过程中, 误差函数 $f$ 与 $\mathrm{B}$ 样条基函数 $\left\{N_{i}(t), i=0,1, \cdots, n\right\}$ 和参数 $\left\{t_{0}<t_{1}<\cdots<t_{m}\right\}$ 也有 关 ${ }^{[17]}$, 通过优化参数和节点可以减小误差 $f$, 从 而提高拟合精度.

由于最小二乘拟合方法需要求解线性方程组, 随着曲线控制顶点的增加, 计算量也会增加, 还可 能产生奇异现象，因此并不适用于大规模数据点 拟合. 下面介绍拟牛顿法中的一种自适应的 BFGS 方法在本文中的应用. 通过计算误差函数关于控 制顶点 $\boldsymbol{P}$ 的梯度, 利用梯度信息对控制顶点进行 迭代调整，以此得到满足精度的拟合曲线.

\section{2 本文方法及收敛性}

\section{1 优化控制顶点}

BFGS 方法将单位矩阵作为初始 Hessian 矩阵 的近似, 利用校正矩阵对 Hessian 矩阵的近似进行 迭代更新. 自适应 BFGS 方法通过对校正矩阵的改 进, 进一步提高了方法的收玫速度.
设误差函数是关于控制顶点 $\boldsymbol{P}$ 为自变量的目 标函数, 记为 $f(\boldsymbol{P})$. 给定一组数据点 $\left\{Q_{j}\right\}_{j=0}^{m}$, 从 中均匀地选择部分数据点作为 $\mathrm{B}$ 样条曲线初始的 控制顶点 $\left\{P_{i}^{0}\right\}_{i=0}^{n}$. 初始数据点对应的参数为 $\left\{0=t_{0}<t_{1}<\cdots<t_{m}=1\right\}$, 得到初始的 B 样条曲线为

$$
C_{0}(t)=\sum_{i=0}^{n} N_{i}(t) P_{i}^{0}=N(t) \boldsymbol{P}^{(0)}, t \in\left[t_{0}, t_{m}\right] .
$$

其中, $\boldsymbol{P}^{(0)}=\left(P_{0}^{0}, P_{1}^{0}, \cdots, P_{n}^{0}\right)^{\mathrm{T}} . f\left(\boldsymbol{P}^{(0)}\right)$ 在 $\boldsymbol{P}^{(0)}$ 处的 梯度为 $\boldsymbol{g}_{0}=\nabla f\left(\boldsymbol{P}^{(0)}\right)=2 \boldsymbol{N}^{\mathrm{T}}\left(\boldsymbol{N} \boldsymbol{P}^{(0)}-\boldsymbol{Q}\right)$.

令 $\boldsymbol{B}_{0}=\boldsymbol{I}_{n+1}$ (单位矩阵), $\boldsymbol{d}_{0}=-\boldsymbol{B}_{0} \boldsymbol{g}_{0}, \boldsymbol{d}_{0}$ 表示 BFGS 方法的搜索方向. 得到第 1 次迭代的控制顶 点为

$$
\boldsymbol{P}^{(1)}=\boldsymbol{P}^{(0)}+\alpha_{0} \boldsymbol{d}_{0}
$$

步长 $\alpha_{0}$ 通过 Wolfe 线性搜索条件确定 ${ }^{[25]}$, 即

$$
\left\{\begin{array}{l}
f\left(\boldsymbol{P}^{0}+\alpha_{0} \boldsymbol{d}_{0}\right) \leqslant f\left(\boldsymbol{P}^{0}\right)+c_{1} \alpha_{0} \boldsymbol{g}\left(\boldsymbol{P}^{0}\right)^{\mathrm{T}} \boldsymbol{d}_{0} \\
\boldsymbol{g}\left(\boldsymbol{P}^{0}+\alpha_{0} \boldsymbol{d}_{0}\right)^{\mathrm{T}} \boldsymbol{d}_{0} \geqslant c_{2} \boldsymbol{g}\left(\boldsymbol{P}^{0}\right)^{\mathrm{T}} \boldsymbol{d}_{0}
\end{array} .\right.
$$

其中, $c_{1}$ 和 $c_{2}$ 表示常数, $0<c_{1}<c_{2}<1$.

由式(3)可以得到迭代 1 次的拟合曲线 $C_{1}(t)$ 为

$$
C_{1}(t)=\boldsymbol{N}(t) \boldsymbol{P}^{(1)}, t \in\left[t_{0}, t_{m}\right] .
$$

其中, $\boldsymbol{P}^{(1)}=\left(P_{0}^{1}, P_{1}^{1}, \cdots, P_{n}^{1}\right)^{\mathrm{T}} . f\left(\boldsymbol{P}^{(1)}\right)$ 在 $\boldsymbol{P}^{(1)}$ 处的梯 度为 $\boldsymbol{g}_{1}=\nabla f\left(\boldsymbol{P}^{(1)}\right)=2 \boldsymbol{N}^{\mathrm{T}}\left(\boldsymbol{N} \boldsymbol{P}^{(1)}-\boldsymbol{Q}\right)$. 可以得到第 2 次迭代的控制顶点为

$$
\boldsymbol{P}^{(2)}=\boldsymbol{P}^{(1)}+\alpha_{1} \boldsymbol{d}_{1}
$$

其中， $\boldsymbol{d}_{1}$ 表示当前 BFGS 方法的搜索方向，即

$$
B_{1} d_{1}=-g_{1}
$$

其中, $\boldsymbol{B}_{1}$ 表示 $\nabla^{2} f\left(\boldsymbol{P}^{(1)}\right)$ 的近似.

BFGS 算法的性能取决于构造的近似矩阵 $\boldsymbol{B}_{k+1}(k=0,1,2, \cdots)$ 的特征值 ${ }^{[26]} .2018$ 年, Andrei ${ }^{[27]}$ 提出一种自适应的 BFGS 方法, 通过加入缩放参 数, 可以有效地对 $\boldsymbol{B}_{k+1}$ 中较大的特征值进行校正, 从而提高算法的性能. 本文利用这种构造方法更 新校正矩阵, 令 $\boldsymbol{s}_{0}=\boldsymbol{P}^{(1)}-\boldsymbol{P}^{(0)}, \boldsymbol{y}_{0}=\boldsymbol{g}_{1}-\boldsymbol{g}_{0}, \gamma_{0}$ 表示缩放 参数, 取值为

$$
\gamma_{0}=\min \left\{\frac{\boldsymbol{y}_{0}^{\mathrm{T}} \boldsymbol{s}_{0}}{\left\|\boldsymbol{y}_{0}\right\|^{2}+\left|\boldsymbol{s}_{0}^{\mathrm{T}} \boldsymbol{g}_{1}\right|}, 1\right\} .
$$

从而得到校正矩阵 


$$
\boldsymbol{B}_{1}=\boldsymbol{B}_{0}-\frac{\boldsymbol{B}_{0} \boldsymbol{s}_{0} \boldsymbol{s}_{0}^{\mathrm{T}} \boldsymbol{B}_{0}}{\boldsymbol{s}_{0}^{\mathrm{T}} \boldsymbol{B}_{0} \boldsymbol{s}_{0}}+\gamma_{0} \frac{\boldsymbol{y}_{0} \boldsymbol{y}_{0}^{\mathrm{T}}}{\boldsymbol{y}_{0}^{\mathrm{T}} \boldsymbol{s}_{0}} .
$$

步长 $\alpha_{1}$ 通过 Wolfe 线性搜索条件确定, 即

$$
\left\{\begin{array}{l}
f\left(\boldsymbol{P}^{(1)}+\alpha_{1} \boldsymbol{d}_{1}\right) \leqslant f\left(\boldsymbol{P}^{(1)}\right)+c_{1} \alpha_{1} \boldsymbol{g}\left(\boldsymbol{P}^{(1)}\right)^{\mathrm{T}} \boldsymbol{d}_{1} . \\
\boldsymbol{g}\left(\boldsymbol{P}^{(1)}+\alpha_{1} \boldsymbol{d}_{1}\right)^{\mathrm{T}} \boldsymbol{d}_{1} \geqslant c_{2} \boldsymbol{g}\left(\boldsymbol{P}^{(1)}\right)^{\mathrm{T}} \boldsymbol{d}_{1}
\end{array}\right.
$$

由式(4)可以得到第 2 次迭代的拟合曲线为

$$
C_{2}(t)=N(t) \boldsymbol{P}^{(2)}, t \in\left[t_{0}, t_{m}\right] .
$$

其中, $\boldsymbol{P}^{(2)}=\left(P_{0}^{2}, P_{1}^{2}, \cdots, P_{n}^{2}\right)^{\mathrm{T}}$.

假设经过 $k$ 次迭代后, 得到的控制顶点为 $\boldsymbol{P}^{(k)}=\left(P_{0}^{k}, P_{1}^{k}, \cdots, P_{n}^{k}\right)^{\mathrm{T}}$ ，相应的拟合曲线表示为 $C_{k}(t), f\left(\boldsymbol{P}^{k}\right)$ 在 $\boldsymbol{P}^{k}$ 处的梯度为

$$
\nabla f\left(\boldsymbol{P}^{(k)}\right)=2 \boldsymbol{N}^{\mathrm{T}}\left(\boldsymbol{N} \boldsymbol{P}^{(k)}-\boldsymbol{Q}\right) .
$$

可以求得 $\boldsymbol{s}_{k}=\boldsymbol{P}^{(k)}-\boldsymbol{P}^{(k-1)}, \boldsymbol{y}_{k}=\boldsymbol{g}_{k}-\boldsymbol{g}_{k-1} \cdot \gamma_{k}$ 表示 第 $k$ 次迭代的缩放参数，取值为

$$
\gamma_{k}=\min \left\{\frac{\boldsymbol{y}_{k}^{\mathrm{T}} \boldsymbol{s}_{k}}{\left\|\boldsymbol{y}_{k}\right\|^{2}+\left|\boldsymbol{s}_{k}^{\mathrm{T}} \boldsymbol{g}_{k}\right|}, 1\right\}
$$

由以上可得第 $k+1$ 次迭代搜索方向为 $\boldsymbol{d}_{k}=-\boldsymbol{H}_{k} \boldsymbol{g}_{k}$, $\boldsymbol{H}_{k}=\boldsymbol{B}_{k}^{-1}$. 其中,

$$
\begin{gathered}
\boldsymbol{H}_{k}=\boldsymbol{H}_{k-1}-\frac{\boldsymbol{H}_{k-1} \boldsymbol{y}_{k} \boldsymbol{s}_{k}^{\mathrm{T}}+\boldsymbol{s}_{k} \boldsymbol{y}_{k}^{\mathrm{T}} \boldsymbol{H}_{k-1}}{\boldsymbol{y}_{k}^{T} \boldsymbol{s}_{k}}+ \\
\left(\frac{1}{\gamma_{k}}+\frac{\boldsymbol{y}_{k}^{\mathrm{T}} \boldsymbol{H}_{k-1} \boldsymbol{y}_{k}}{\boldsymbol{y}_{k}^{\mathrm{T}} \boldsymbol{s}_{k}}\right) \frac{\boldsymbol{s}_{k} \boldsymbol{s}_{k}^{\mathrm{T}}}{\boldsymbol{y}_{k}^{\mathrm{T}} \boldsymbol{s}_{k}} .
\end{gathered}
$$

步长 $\alpha_{k}$ 通过 Wolfe 线性搜索条件确定, 即

$$
\left\{\begin{array}{l}
f\left(\boldsymbol{P}^{k}+\alpha_{k} \boldsymbol{d}_{k}\right) \leqslant f\left(\boldsymbol{P}^{k}\right)+c_{1} \alpha_{k} \boldsymbol{g}\left(\boldsymbol{P}^{k}\right)^{\mathrm{T}} \boldsymbol{d}_{k} \\
\boldsymbol{g}\left(\boldsymbol{P}^{k}+\alpha_{k} \boldsymbol{d}_{k}\right)^{\mathrm{T}} \boldsymbol{d}_{k} \geqslant c_{2} \boldsymbol{g}\left(\boldsymbol{P}^{k}\right)^{\mathrm{T}} \boldsymbol{d}_{k}
\end{array} .\right.
$$

类似地, 可以得到第 $k+1$ 次迭代的控制顶点

$$
\boldsymbol{P}^{(k+1)}=\boldsymbol{P}^{(k)}+\alpha_{k} \boldsymbol{d}_{k}
$$

由式(6)可以得到 $k+1$ 次迭代的拟合曲线为

$$
C_{k+1}(t)=\boldsymbol{N}(t) \boldsymbol{P}^{(k+1)}, t \in\left[t_{0}, t_{m}\right] .
$$

其中, $\boldsymbol{P}^{(k+1)}=\left(P_{0}^{k+1}, P_{1}^{k+1}, \cdots, P_{n}^{k+1}\right)^{\mathrm{T}}$.

为了保证迭代过程中拟合曲线始终插值首末 端点 $Q_{0}$ 和 $Q_{m}$, 令 $P_{0}^{0}=Q_{0}, P_{n}^{0}=Q_{m}, P_{0}^{k+1}=P_{0}^{k}$, $P_{n}^{k+1}=P_{n}^{k}, k=0,1,2, \cdots$.

\section{2 优化数据点参数}

通过自适应 BFGS 方法对控制顶点进行调整，
可以得到一组拟合曲线, 但是数据点与曲线上对 应参数点之间的距离不是最短的. 通过对数据点 参数的优化, 可以减少两者的距离, 进一步减小拟 合误差.

本节将参数值作为影响拟合误差的自变量, 为了保证优化后的参数值是按序递增的, 通过步 长加速法 ${ }^{[28]}$ 对其进行优化. 步长加速法是一种不 需要计算梯度的直接方法, 它适用于任何形式的 目标函数，编程简单. 该方法主要由 2 部分组成, 通过探索搜索寻找当前迭代点的下降方向，再通 过模式移动沿着这个有利的方向寻找新的迭代点.

设此时目标函数为 $f(\boldsymbol{t}), \boldsymbol{t}^{(0)}=\left(t_{0}^{(0)}, t_{1}^{(0)}, \cdots, t_{m}^{(0)}\right)^{\mathrm{T}}$ 表示初始的参数向量. 由于首末的参数值是固定 的, 所以实际是从第 2 个参数值进行逐个优化. 设 优化参数为 $t_{j}^{(0)}, j=1,2, \cdots, m-1$. 初始化每个参数沿 坐标方向探查长度 $\delta_{j}$, 步长收缩系数 $\omega=0.5, c=1$, 预先设定步长收缩系数阈值 $\varepsilon$, 令 $t_{\mathrm{L} j}^{(0)}=t_{j}^{(0)}-c \delta_{j}$, $t_{\mathrm{R} j}^{(0)}=t_{j}^{(0)}+c \delta_{j}$.

若拟合误差 $f\left(t_{\mathrm{Rj}}^{(0)}\right) \geqslant f\left(t_{j}^{(0)}\right), f\left(t_{\mathrm{L} j}^{(0)}\right) \geqslant f\left(t_{\mathrm{Rj}}^{(0)}\right)$; 则说明探测搜索失败, 缩短给定的步长. 令 $c=\omega c$, 则 $t_{\mathrm{L} j}^{(0)}=t_{j}^{(0)}-\omega c \delta_{j}, t_{\mathrm{R} j}^{(0)}=t_{j}^{(0)}+\omega c \delta_{j}$, 直至步长收 缩系数小于阈值 $\varepsilon$. 若拟合误差 $f\left(t_{\mathrm{L} j}^{(0)}\right)<f\left(t_{j}^{(0)}\right)$, 取 $d_{j}^{(0)}=t_{\mathrm{L} j}^{(0)}$; 若拟合误差 $f\left(t_{\mathrm{R} j}^{(0)}\right)<f\left(t_{j}^{(0)}\right) \leqslant f\left(t_{\mathrm{L} j}^{(0)}\right)$, 取 $d_{j}^{(0)}=t_{\mathrm{R} j}^{(0)}$, 做模式移动, 即 $t_{j}^{(1)}=t_{j}^{(0)}+\left(t_{j}^{(0)}-d_{j}^{(0)}\right)$. 其中, $t_{j}^{(1)}$ 作为新的参考点, $d_{j}^{(0)}$ 作为新的基点, 新的拟合误差为 $f\left(t_{j}^{(1)}\right)$. 令 $t_{\mathrm{L} j}^{(1)}=t_{j}^{(1)}-c \delta_{j}, t_{\mathrm{R} j}^{(1)}=$; $t_{j}^{(1)}+c \delta_{j}$; . 若拟合误差增大, 则说明探测搜索失 败. 令 $c=\omega c$, 则 $t_{\mathrm{L} j}^{(1)}=t_{j}^{(1)}-\omega c \delta_{j}, t_{\mathrm{R} j}^{(1)}=t_{j}^{(1)}+\omega c \delta_{j}$. 直至步长收缩系数小于阈值 $\varepsilon$. 若拟合误差 $f\left(t_{\mathrm{L} j}^{(1)}\right)<f\left(t_{j}^{(1)}\right)$, 取 $d_{j}^{(1)}=t_{\mathrm{L} j}^{(1)}$; 若拟合误差 $f\left(t_{\mathrm{Rj}}^{(1)}\right)<f\left(t_{j}^{(1)}\right) \leqslant f\left(t_{\mathrm{L} j}^{(1)}\right)$, 取 $d_{j}^{(1)}=t_{\mathrm{Rj}}^{(1)}$, 做模式移 动, 即 $t_{j}^{(2)}=t_{j}^{(1)}+\left(d_{j}^{(1)}-d_{j}^{(0)}\right)$; 其中, $d_{j}^{(1)}$ 作为新的 基点.

依此类推，设第 $k$ 次迭代的拟合误差为 $f\left(t_{j}^{(k)}\right)$, 令 $t_{\mathrm{L} j}^{(k)}=t_{j}^{(k)}-c \delta_{j}, t_{\mathrm{R} j}^{(k)}=t_{j}^{(k)}+c \delta_{j}$; 若拟合误差增大, 则说明探测搜索失败. 令 $c=\omega c, t_{\mathrm{L} j}^{(k)}=t_{j}^{(k)}-\omega c \delta_{j}$; $t_{\mathrm{Rj}}^{(k)}=t_{j}^{(k)}+\omega c \delta_{j}$, 直至步长收缩系数小于國值 $\varepsilon$. 
若拟合误差 $f\left(t_{\mathrm{L} j}^{(k)}\right)<f\left(t_{j}^{(k)}\right)$, 取 $d_{j}^{(k)}=t_{\mathrm{L} j}^{(k)}$; 若拟合 误差 $f\left(t_{\mathrm{R} j}^{(k)}\right)<f\left(t_{j}^{(k)}\right) \leqslant f\left(t_{\mathrm{L} j}^{(k)}\right)$, 取 $d_{j}^{(k)}=t_{\mathrm{R} j}^{(k)}$, 做模 式移动, 即 $t_{j}^{(k+1)}=t_{j}^{(k)}+\left(d_{j}^{(k)}-d_{j}^{(k-1)}\right)$. 当步长收 缩系数小于阈值 $\varepsilon$ 或到达给定的迭代次数时，优 化下一个数据点对应的参数值.

当所有的参数都进行优化后, 循环结束, 得到 一组优化后的参数值. 再利用本文迭代控制顶点 的方法，重新得到新的拟合曲线.

\section{3 算法流程及收敛性}

当缩放参数选择式(5)时，文献[27]证明了自 适应 BFGS 方法的收玫性，本文利用文献[27]的方 法，证明了本文方法的收敛性.

假设 $\boldsymbol{P}^{k}$ 是由第 2.1 节产生的一组控制点, $f(\boldsymbol{P})$ 是连续可微函数, 有界水平集 $D=\{\boldsymbol{P} \mid f(\boldsymbol{P}) \leqslant$ $\left.f\left(\boldsymbol{P}^{(0)}\right)\right\}$ 是凸集, 给定初始点为 $\boldsymbol{P}^{(0)}=\left(P_{0}^{0}, P_{1}^{0}, \cdots\right.$, $\left.P_{n}^{0}\right)^{\mathrm{T}}$, 由文献 [27]可知 $\boldsymbol{g}_{k}$ 满足 $\liminf _{k \rightarrow \infty}\left\|\boldsymbol{g}_{k}\right\|=0$, 由于

$$
\begin{aligned}
& \boldsymbol{g}_{k}=\nabla f\left(\boldsymbol{P}^{(k)}\right), \\
& \nabla f\left(\boldsymbol{P}^{k}\right)=2 \boldsymbol{N}^{\mathrm{T}}\left(\boldsymbol{N} \boldsymbol{P}^{k}-\boldsymbol{Q}\right), \\
& \nabla f\left(\boldsymbol{P}^{\infty}\right)=2 \boldsymbol{N}^{\mathrm{T}} \boldsymbol{N} \boldsymbol{P}^{\infty}-2 \boldsymbol{N}^{\mathrm{T}} \boldsymbol{Q}=0 ;
\end{aligned}
$$

因此 $\left(\boldsymbol{N}^{\mathrm{T}} \boldsymbol{N}\right) \boldsymbol{P}^{\infty}=\boldsymbol{N}^{\mathrm{T}} \boldsymbol{Q}$. 所以本文调整控制顶点 的方法是收玫的，且极限曲线为最小二乘拟合结 果. 由于目标函数 $f(\boldsymbol{t})$ 是可微的, 因此步长加速 法对数据点参数的优化是收玫的, 其收玫速度是 线性的.

本文方法流程如图 1 所示. 本文利用自适应 BFGS 方法优化控制顶点，在前几次迭代中收敛较 快. 当迭代达到一定次数后, 拟合误差变化较小, 此时保持控制顶点不变, 利用步长加速法对数据 点参数进行优化调整, 得到新的数据点参数. 再将 其代人自适应 BFGS 控制顶点迭代中，得到新的拟 合曲线.

但是，本文在迭代过程中利用步长加速法对 数据点参数进行优化时, 初始探查长度是人为设 定的. 当初始探查长度设定偏大时, 调整步长的次 数会增加, 从而增加计算时间. 而初始探查长度设 定偏小时, 优化后的参数可能是局部最优解. 由于 步长加速法的收玫速度是线性的, 虽然可以收玫 到平稳点, 但收玫速度较慢.

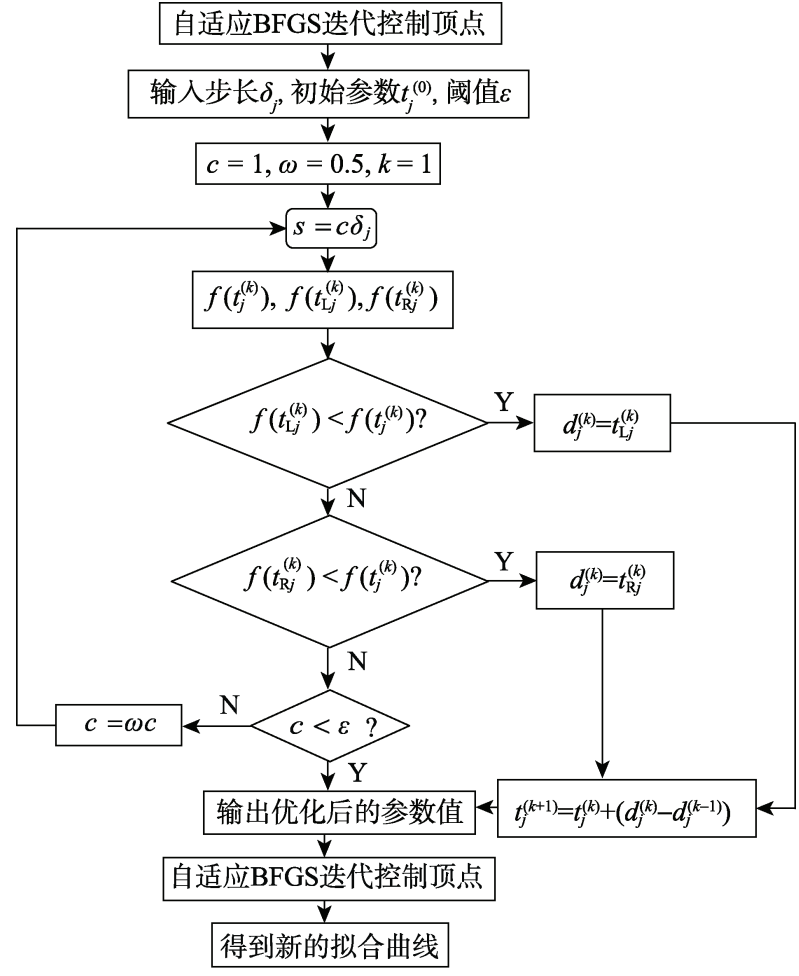

图 1 本文方法流程

\section{3 数值实例}

\section{1 方法准备}

本节以三次 $\mathrm{B}$ 样条曲线为例拟合给定的数据点, 在配置为 $2.2 \mathrm{GHz}$ 英特尔双核处理器, 内存 $8 \mathrm{~GB}$ 的 计算机上, 采用 MATLAB 编程实现本文方法. 初始 的数据点参数 $\left\{t_{j}\right\}_{j=0}^{m}$ 用弦长参数法 ${ }^{[29]}$ 确定, 即

$$
\begin{aligned}
& t_{0}=0, \quad t_{1}=1, \\
& t_{j}=t_{j-1}+\frac{\left\|Q_{j}-Q_{j-1}\right\|}{\sum_{j=0}^{m}\left\|Q_{j}-Q_{j-1}\right\|}, j=1,2, \cdots, m .
\end{aligned}
$$

本文节点向量定义为

$$
\left\{0,0,0,0, u_{4}^{0}, u_{5}^{0}, \cdots, u_{n}^{0}, 1,1,1,1\right\} .
$$

其中, $u_{j+3}=(1-\alpha) t_{i-1}+\alpha t_{i}, j=1, \cdots, n-3, i=[j d]$, $\alpha=j d-i, d=\frac{m+1}{n-2}$. [ $\left.j d\right]$ 表示不超过 $j d$ 的最大 整数.

\section{2 方法实例}

本节通过对一些平面图形随机采点，以 4 个例 子展示了本文方法的有效性. 第 1 个例子是火焰 (552 个数据点), 第 2 个例子是美人鱼 (454 个数据 点), 第 3 个例子是枝头鸟 (896 个数据点), 第 4 个 
例子是凤凰(1849 个数据点), 控制点的数目分别 选择为 90 个, 100 个, 250 个, 450 个.

由于本文调整控制顶点的方法也收敛到最小 二乘的拟合结果，在相同条件下与文献[10-12]的 方法进行了比较. 图 2 图 5 展示本文方法对不同 数据的拟合曲线效果, 以及与其他 3 种方法的误差 对比图; 图 2c 图 5c 中黑线表示未经参数优化的 拟合曲线，红线表示经过参数优化的拟合曲线. 从 数值实例中的局部细节放大图可以看出, 对数据

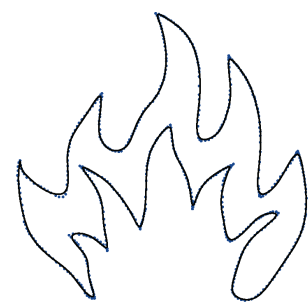

a. 本文方法迭代5次拟合效果

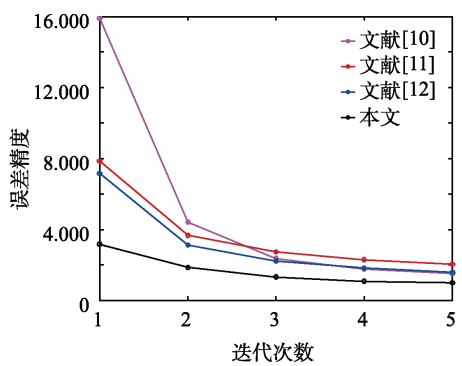

b. 误差对比图

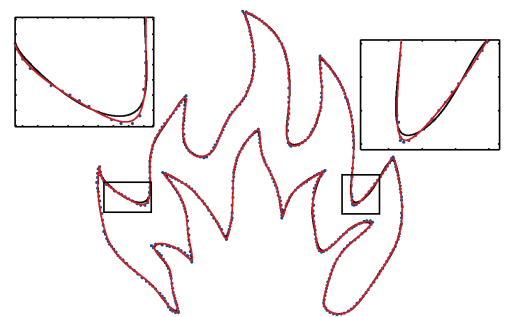

c. 最终拟合曲线和局部细节放大图

图 2 本文方法拟合火焰轮廓线及误差

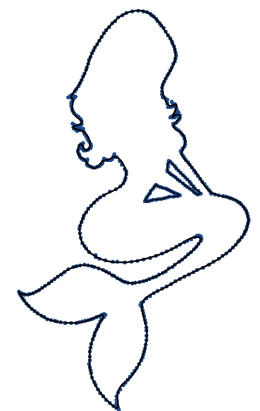

a. 本文方法迭代5次拟合效果

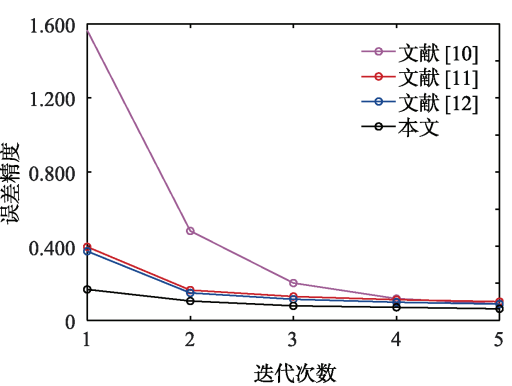

b. 误差对比图

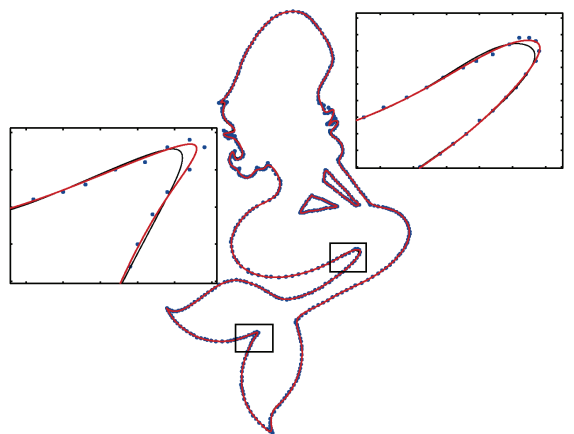

c. 最终拟合曲线和局部细节放大图

图 3 本文方法拟合美人鱼轮廓线及误差
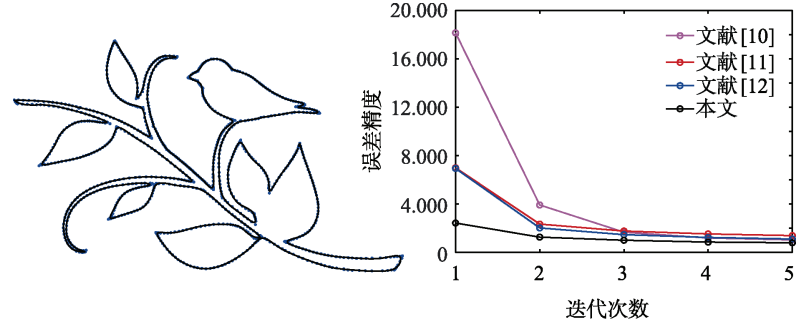

a. 本文方法迭代5次拟合效果

b. 误差对比图

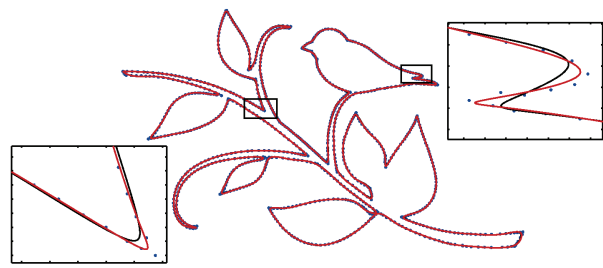

c. 最终拟合曲线和局部细节放大图

图 4 本文方法拟合枝头鸟轮廓线及误差

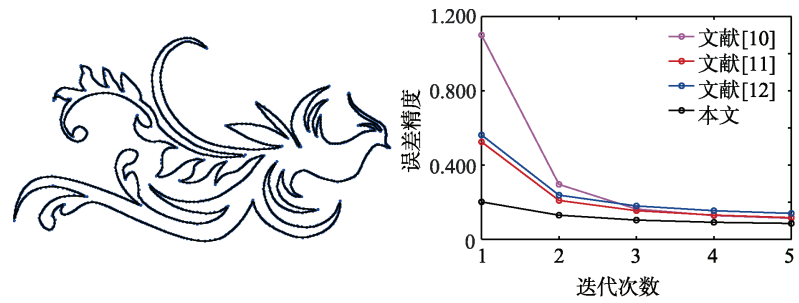

a. 本文方法迭代5次拟合效果

b. 误差对比图

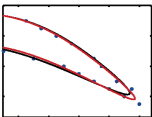

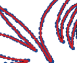
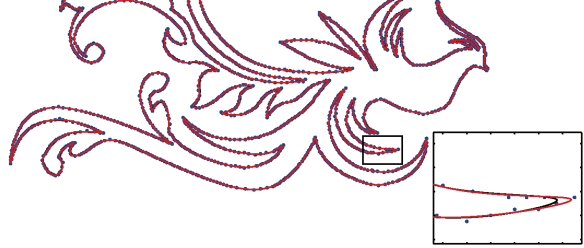

c. 最终拟合曲线和局部细节放大图

图 5 本文方法拟合凤凰轮廓线及误差

点参数优化后的拟合曲线比未优化后的拟合曲线 视觉效果更好, 更加逼近给定的数据点. 表 1 表 4 分别列出了 4 种方法拟合采样点的误差比较.

表 14 种方法拟合火焰轮廓线采样点的误差

\begin{tabular}{cllll}
\hline 迭代次数 & 文献[10] & 文献[11] & 文献[12] & 本文 \\
\hline 0 & $1.247 \times 10^{2}$ & $1.247 \times 10^{2}$ & $1.247 \times 10^{2}$ & $1.247 \times 10^{2}$ \\
1 & $1.590 \times 10^{1}$ & 7.859 & 7.160 & 3.171 \\
2 & 4.419 & 3.659 & 3.120 & 1.855 \\
3 & 2.371 & 2.745 & 2.233 & 1.326 \\
4 & 1.779 & 2.302 & 1.817 & 1.084 \\
5 & 1.513 & 2.035 & 1.571 & $9.908 \times 10^{-1}$ \\
\hline
\end{tabular}


表 24 种方法拟合美人鱼轮廓线采样点的误差

\begin{tabular}{cllll}
\hline 迭代次数 & 文献[10] & 文献[11] & 文献[12] & 本文 \\
\hline 0 & 7.348 & 7.348 & 7.348 & 7.348 \\
1 & 1.565 & $3.963 \times 10^{-1}$ & $3.727 \times 10^{-1}$ & $1.666 \times 10^{-1}$ \\
2 & $4.825 \times 10^{-1}$ & $1.631 \times 10^{-1}$ & $1.478 \times 10^{-1}$ & $1.041 \times 10^{-1}$ \\
3 & $2.014 \times 10^{-1}$ & $1.283 \times 10^{-1}$ & $1.137 \times 10^{-1}$ & $7.810 \times 10^{-2}$ \\
4 & $1.170 \times 10^{-1}$ & $1.114 \times 10^{-1}$ & $9.76 \times 10^{-2}$ & $7.006 \times 10^{-2}$ \\
5 & $8.813 \times 10^{-2}$ & $1.007 \times 10^{-1}$ & $8.75 \times 10^{-2}$ & $6.225 \times 10^{-2}$ \\
\hline
\end{tabular}

表 34 种方法拟合枝头鸟轮廓线采样点的误差

\begin{tabular}{cllll}
\hline 迭代次数 & 文献[10] & 文献[11] & 文献[12] & 本文 \\
\hline 0 & $1.428 \times 10^{2}$ & $1.428 \times 10^{2}$ & $1.428 \times 10^{2}$ & $1.428 \times 10^{2}$ \\
1 & $1.814 \times 10^{1}$ & 6.986 & 6.934 & 2.427 \\
2 & 3.915 & 2.336 & 2.019 & 1.260 \\
3 & 1.669 & 1.764 & 1.461 & 1.000 \\
4 & 1.191 & 1.524 & 1.235 & $8.516 \times 10^{-1}$ \\
5 & 1.034 & 1.383 & 1.104 & $7.958 \times 10^{-1}$ \\
\hline
\end{tabular}

表 44 种方法拟合凤凰轮廓线采样点的误差

\begin{tabular}{cllll}
\hline 迭代次数 & 文献[10] & 文献[11] & 文献[12] & 本文 \\
\hline 0 & 8.979 & 8.979 & 8.979 & 8.979 \\
1 & 1.100 & $5.242 \times 10^{-1}$ & $5.615 \times 10^{-1}$ & $2.009 \times 10^{-1}$ \\
2 & $2.951 \times 10^{-1}$ & $2.088 \times 10^{-1}$ & $2.373 \times 10^{-1}$ & $1.294 \times 10^{-1}$ \\
3 & $1.621 \times 10^{-1}$ & $1.536 \times 10^{-1}$ & $1.790 \times 10^{-1}$ & $1.030 \times 10^{-1}$ \\
4 & $1.274 \times 10^{-1}$ & $1.297 \times 10^{-1}$ & $1.535 \times 10^{-1}$ & $9.093 \times 10^{-2}$ \\
5 & $1.129 \times 10^{-1}$ & $1.161 \times 10^{-1}$ & $1.390 \times 10^{-1}$ & $8.490 \times 10^{-2}$ \\
\hline
\end{tabular}

表 5 给出了参数的优化次数对拟合曲线误差 的影响，经过 5 次迭代后，固定上述控制顶点，利 用步长加速法对数据点参数分别进行 5 次、 10 次、 20 次优化后的误差. 优化次数为 0 表示控制顶点 迭代 5 次而数据点参数未经优化.

表 5 参数优化次数对拟合曲线误差的影响

\begin{tabular}{ccccc}
\hline \multirow{2}{*}{ 优化次数 } & \multicolumn{4}{c}{ 轮廓线 } \\
\cline { 2 - 5 } & 火焰 & 美人鱼 & 枝头鸟 & 凤凰 \\
\hline 0 & $1.772 \times 10^{-1}$ & $3.269 \times 10^{-2}$ & $4.852 \times 10^{-1}$ & $3.621 \times 10^{-2}$ \\
5 & $7.513 \times 10^{-2}$ & $1.257 \times 10^{-2}$ & $1.598 \times 10^{-1}$ & $1.774 \times 10^{-2}$ \\
10 & $4.841 \times 10^{-2}$ & $8.663 \times 10^{-3}$ & $1.146 \times 10^{-1}$ & $1.338 \times 10^{-2}$ \\
20 & $3.727 \times 10^{-2}$ & $6.735 \times 10^{-3}$ & $9.760 \times 10^{-2}$ & $7.273 \times 10^{-3}$ \\
\hline
\end{tabular}

\section{4 结 语}

本文优化控制顶点的方法在前几次迭代中收 玫较快，因此适用于对时间成本要求较高但对误 差精度要求较低的情形. 此外, 与现有的 3 种数据
点拟合方法对比，本文方法在前几次迭代的收玫 速度更快. 利用步长加速法对每个数据点的参数 进行优化, 保证了参数向量的有序性, 提高了曲线 的拟合精度，使其更加逼近给定的数据点.

\section{参考文献(References):}

[1] Mudur S P. Mathematical elements for computer graphics[M] // Advances in Computer Graphics. Heidelberg: Springer, 1986: 53-134

[2] Pottmann H, Leopoldseder S, Hofer M, et al. Industrial geometry: recent advances and applications in $\mathrm{CAD}[\mathrm{J}]$. Computer-Aided Design, 2005, 37(7): 751-766

[3] Hoschek J, Lasse D, Schumaker L L. Fundamentals of computer aided geometric design[M]. Natick MA: AK Peters, Ltd, 1993

[4] Qi Dongxu, Tian Zixian, Zhang Yuxin, et al. The method of numeric polish in curve fitting $[\mathrm{J}]$. Acta Mathematica Sinica, 1975, 18(3): 173-184(in Chinese)

(齐东旭, 田自贤, 张玉心, 等. 曲线拟合的数值磨光方法 [J]. 数学学报, 1975, 18(3): 173-184)

[5] de Boor C. How does Agee's smoothing method work?[R]. Washington D C: Army Research Office 79-3, 1979: 299-302

[6] Lin Hongwei, Wang Guojin, Dong Chenshi. Constructing iterative non-uniform B-spline curve and surface to fit data points[J]. Science in China: Series E, 2003, 33(10): 912-923(in Chinese)

(萄宏伟, 王国瑾, 董辰世. 用迭代非均匀 B-spline 曲线(曲 面)拟合给定点集 [J]. 中国科学: E 辑, 2003, 33(10): 912-923)

[7] Lin H W, Bao H J, Wang G J. Totally positive bases and progressive iteration approximation[J]. Computers \& Mathematics with Applications, 2005, 50(3/4): 575-586

[8] $\mathrm{Lu} \mathrm{L} \mathrm{Z}$. Weighted progressive iteration approximation and convergence analysis $[\mathrm{J}]$. Computer Aided Geometric Design, 2010, 27(2): 129-137

[9] Lin H W, Zhang Z Y. An extended iterative format for the progressive-iteration approximation[J]. Computers \& Graphics, 2011, 35(5): 967-975

[10] Deng C Y, Lin H W. Progressive and iterative approximation for least squares B-spline curve and surface fitting[J]. Computer-Aided Design, 2014, 47: 32-44

[11] Zhang L, Ge X Y, Tan J Q. Least square geometric iterative fitting method for generalized B-spline curves with two different kinds of weights[J]. The Visual Computer, 2016, 32(9): 1109-1120

[12] Zhang L, Tan J Q, Ge X Y, et al. Generalized B-splines' geometric iterative fitting method with mutually different weights[J]. Journal of Computational and Applied Mathematics, 2018, 329: 331-343

[13] Maekawa T, Matsumoto Y, Namiki K. Interpolation by geometric algorithm[J]. Computer-Aided Design, 2007, 39(4): 313-323

[14] Lin Hongwei. Survey on geometric iterative methods with applications[J]. Journal of Computer-Aided Design \& Computer Graphics, 2015, 27(4): 582-589(in Chinese) 
(萄宏伟. 几何迭代法及其应用综述 $[\mathrm{J}]$. 计算机辅助设计与 图形学学报, 2015, 27(4): 582-589)

[15] Lin H W, Maekawa T, Deng C Y. Survey on geometric iterative methods and their applications[J]. Computer-Aided Design, 2018, 95: 40-51

[16] Speer T, Kuppe M, Hoschek J. Global reparametrization for curve approximation[J]. Computer Aided Geometric Design, 1998, 15(9): 869-877

[17] Zhou Minghua, Wang Guozhao. Genetic algorithm-based least square fitting of B-spline and Bézier curves[J]. Journal of Computer Research and Development, 2005, 42(1): 134-143(in Chinese)

(周明华, 汪国昭. 基于遗传算法的 B 样条曲线和 Bézier 曲 线的最小二乘拟合 [J]. 计算机研究与发展，2005，42(1): 134-143)

[18] Hu L C, Shou H H, Fang S F. A PIA optimization algorithm for non-uniform B-spline curves and surfaces[J]. International Journal of Modelling and Simulation, 2017, 37(3): 167-177

[19] Zhang Meng, Li Yajuan, Deng Chongyang. Optimizing NURBS curves fitting by least squares progressive and iterative approximation[J]. Journal of Computer-Aided Design \& Computer Graphics, 2020, 32(4): 568-574(in Chinese)

(张蒙, 李亚娟, 邓重阳. NURBS 曲线拟合的最小二乘渐进 迭代逼近优化算法 [J]. 计算机辅助设计与图形学学报, 2020, 32(4): 568-574)

[20] Li Shasha, Xu Huixia, Deng Chongyang. Data-weighted least square progressive and iterative approximation and related
B-spline curve fitting[J]. Journal of Computer-Aided Design \& Computer Graphics, 2019, 31(9): 1574-1580(in Chinese)

(李莎莎, 徐惠霞, 邓重阳. 数据点加权最小二乘渐进迭代 逼近及其 $\mathrm{B}$ 样条曲线拟合 $[\mathrm{J}]$. 计算机辅助设计与图形学学 报, 2019, 31(9): 1574-1580)

[21] Broyden C G. The convergence of a class of double-rank minimization algorithms 1. general considerations[J]. IMA Journal of Applied Mathematics, 1970, 6(1): 76-90

[22] Fletcher R. A new approach to variable metric algorithms[J]. The Computer Journal, 1970, 13(3): 317-322

[23] Goldfarb D. A family of variable metric methods derived by variational means[J]. Mathematics of Computation, 1970, 24(109): $23-26$

[24] Shanno D F. Conditioning of quasi-Newton methods for function minimization[J]. Mathematics of Computation, 1970, 24(111): 647-656

[25] Nocedal J, Wright S J. Numerical optimization[M]. 2nd ed. Heidelberg: Springer, 1999: 37-41

[26] Nocedal J. Theory of algorithms for unconstrained optimization[J]. Acta Numerica, 1992, 1: 199-242

[27] Andrei N. An adaptive scaled BFGS method for unconstrained optimization[J]. Numerical Algorithms, 2018, 77(2): 413-432

[28] Hooke R, Jeeves T A. Direct search solution of numerical and statistical problems[J]. Journal of the ACM, 1961, 8(2): 212-229

[29] Piegl L, Tiller W. The NURBS book[M]. 2nd ed. Heidelberg: Springer, 1997: 52-77 